Scholars International Journal of Biochemistry

Abbreviated Key Title: Sch Int J Biochem ISSN 2616-8650 (Print) |ISSN 2617-3476 (Online) Scholars Middle East Publishers, Dubai, United Arab Emirates Journal homepage: https://scholarsmepub.com/sijb/

\title{
Cancer Ovary and Early Diagnosis
}

\author{
Dr. Anil Batta
}

Professor \& Head Department of Medical Biochemistry Govt. Medical College, Amritsar Punjab India

DOI: $10.36348 /$ SIJB.2019.v02i09.001

| Received: 26.08.2019 | Accepted: 16.09.2019 | Published: 29.09.2019

*Corresponding author: Dr. Anil Batta

\section{Abstract}

Between all of the gynecological cancers, ovarian cancer, despite medical advances and the development of diagnostic tools such as biomarkers and detection techniques, remains a fatal cancer with high progression. Despite this, there is no effective screening strategy or standard treatment for ovarian cancer. If diagnosed during stage I, ovarian cancer has a $90 \%$ 5-year survival rate; however, there is usually a masking of symptoms which leads to an often late-stage diagnosis and correspondingly poor survival rate. Current diagnostic methods are invasive and consist of a pelvic examination, transvaginal ultrasonography, and blood tests to detect cancer antigen 125 (CA125). Unfortunately, surgery is often still required to make a positive diagnosis. Epithelial Ovarian Cancer (EOC) is the most common, whereas, stromal and germ cell tumors are of lower abundance. A Risk of Ovarian Malignancy Algorithm (ROMA) classifies patients as being at low or high risk for malignant disease using both the CA125 and HE4 results and a woman's menopausal status. The ROMA index was calculated according to the levels of HE4 and CA-125. HE4 and CA-125 values were input to the ovarian cancer risk assessment software, followed by automatic calculation of the corresponding ROMA index. The premenopausal calculation formula of the ROMA index was: $12+2.38 \times$ LN (HE4) $+0.0626 \times$ LN (CA-125). The postmenopausal calculation formula of the ROMA index was: $8.09+1.04 \times$ LN $(\mathrm{HE} 4)+0.732 \times \mathrm{LN}(\mathrm{CA}-125)$. Such diagnostic medical methods and biomarkers include vaginal and pelvic examination, diagnostic imaging, serum CA125, and screening tests or a combination used in medical centers, however, it is necessary to find new biomarkers with longterm stability and high specificity and sensitivity to detect Ovarian Ca in early stages of disease.

Keywords: Ovarian Cancer; Serum and genetic Biomarkers; Early diagnosis.

Copyright @ 2019: This is an open-access article distributed under the terms of the Creative Commons Attribution license which permits unrestricted use, distribution, and reproduction in any medium for non-commercial use (NonCommercial, or CC-BY-NC) provided the original author and source are credited.

\section{OBJECTIVES}

Evaluate the diagnostic accuracy of serum cancer antigen 125 (CA125) and human epididymis protein 4 (HE4) in prediction of malignant ovarian masses.

\section{STUDY DESIGN}

This prospective study was performed in Govt. Medical College, Amritsar. The eligibility criteria for inclusion were; consecutive women, at any age $\geq 18$ years, with established diagnosis of ovarian mass based on symptoms, signs, and imaging techniques. All patients underwent personal and medical history taking, preoperative serum CA125 and HE4 (cutoff $35 \mathrm{IU} / \mathrm{mL}$ and $150 \mathrm{pmol} / \mathrm{L}$, respectively) assessment then postoperative histopathologic examination of lesions as a reference standard.

\section{RESULTS}

Among the included 100 patients, 54 were confirmed to have ovarian malignancy and 46 had benign lesions. Along with 100 healthy individuals accompanying the patients to the hospital were included. Serum CA125 $\geq 35 \mathrm{IU} / \mathrm{mL}$ was associated with ovarian malignancy at sensitivity $91.9 \%$, specificity $53.8 \%$ and accuracy $70.7 \%$. Raising its cutoff to $67.5 \mathrm{IU} / \mathrm{mL}$ decreased the sensitivity $83.9 \%$, increased the specificity $80.7 \%$ with accuracy $82.1 \%$. The combination of HE4 and CA125 showed sensitivity $75.8 \%$, specificity $93.5 \%$, and accuracy $85.7 \%$. Women suffering from both diabetes mellitus and hypertension showed a significant decrease in CA125 concentration $\mathrm{P}=0.02$ with false negative results in $(5 / 11)$ of them, making its sensitivity $54.5 \%$ in this condition. A Risk of Ovarian Malignancy Algorithm (ROMA) classifies patients as being at low or high risk for malignant disease using both the CA125 and HE4 results and a woman's menopausal status. The serum levels of HE4, CA-125 and ROMA index in the ovarian cancer group were significantly higher than those in the benign tumor and healthy control groups, and there was significant difference $(\mathrm{P}<0.05)$. The expression level of HE4 and ROMA index in the benign tumor group was not 
significantly different. The expression level of CA-125 in serum was significantly higher than that in the healthy control group $(\mathrm{P}<0.05$, Table I).

\section{CONCLUSIONS}

The performance of CA125 in cancer ovary prediction can be improved by increasing its cutoff or by combining CA125 with HE4. Diabetes mellitus and hypertension can influence CA125 performance while HE4 is independent on these factors. This can be an additional value of the introduction of HE4 in cancer ovary prediction protocols.

\section{INTRODUCTION}

Ovarian cancer is the fifth frequency occurring cancer among women and the leading cause of death among gynecological cancers. Malignant epithelial ovarian tumors account for $90 \%$ of all malignancies of the ovary and are the fourth most common cause of tumor-related death in women. The empirical lifetime risk of developing ovarian cancer is 1:70 [1], and most women present with advanced disease (FIGO stage III or stage IV), which is rarely curable. Tumor-associated antigens released into the circulation have been described in many diseases. Ideally, a tumor marker should be able to detect subclinical disease (i.e., screening), useful in monitoring the response to treatment, and to identify early recurrence so that further treatment can be instituted. Furthermore, the release of circulating tumor antigen provides an identifiable surface target on the tumor cell that might be used for in vivo diagnosis or antigen directed therapy [11]. No serum tumor marker, with the possible exception of human $\beta$ chorionic gonadotrophin, meets all these criteria. Nevertheless, measurement of many serum tumor markers has been incorporated into clinical practice. This review will focus on CA125, the most clinically applicable tumor marker for ovarian cancer, and will briefly describe other tumor markers and possible future applications of tumor markers.

\section{Screening and Diagnosis}

As early-stage ovarian cancer carries a much more favorable prognosis, there is an urgent need to identify subclinical disease. A satisfactory method of screening subclinical disease for ovarian cancer is needed. Serological markers are theoretically an ideal approach but none have $100 \%$ specificity and sensitivity.

Population screening with ultrasonography alone has not proved to be a cost-effective means of detecting ovarian cancer. However, the sensitivity and specificity of this investigation can be increased by transvaginal ultrasonography and transvaginal color Doppler imaging. Serum CA125 measurement in healthy women has been used as a means of selecting women for ultrasonography. This increases the specificity of examination, but the predictive value of screening is about $10 \%$.

The inclusion of other tumor markers may further increase the specificity of screening. Einhorn et al. evaluated CA125 concentrations together with those of CA15-3 and TAG-72 in 219 patients undergoing diagnostic laparotomy for pelvic masses. They found that the three tumor markers increased the specificity for detecting ovarian cancer but reduced the sensitivity of the CA125 assay [10]. One such protein is osteopontin, a glycophosphoprotein secreted by activated T lymphocytes, macrophages and leukocytes, found in extracellular matrix, sites of inflammation and body fluids. The gene encoding HE4 is commonly amplified in ovarian tumors. While the exact function of HE4 remains uncharacterized, it is a secreted protein that is absent in normal ovarian surface epithelium, but expressed specifically in $100 \%$ of the 16 human endometrioid epithelial ovarian cancers screened and $93 \%$ of the 60 serous ovarian carcinomas stained for HE4. VEGF levels have been known to be elevated in ovarian cancer patients, where it contributes to the accumulation of ascites. CA19-9 levels typically have mucinous tumors, whereas CA125 is frequently less elevated in these patients, and therefore, CA19-9 levels could be a useful biomarker for this histotype[4]. However, there have not been enough studies, or studies with enough patients, at present, to determine whether CA19-9 is a reliable biomarker for $\mathrm{OC}$, and these studies still require the invasive collection of patient serum.

\section{Monitoring Response to Therapy}

The use of tumor markers to monitor response to treatment is particularly helpful in ovarian cancer where there is often a lack of clinically or radiologically measurable disease. A reduction in the serum CA125 level correlates well with clinical response. Failure of CA125 to fall with chemotherapy indicates drug resistance and identifies a need to change treatment.

\section{Relapse}

It has been accepted for a long time that a rise in CA125 into the abnormal range is highly predictive for relapse $[3,5]$. However, the lead time to clinical relapse is variable and a clearer definition of relapse is needed if CA125 measurement is to be used as a definition of clinical progression. Measurements of CA125 are frequently taken after the completion of chemotherapy, outside clinical trials. From the results of Rustin et al. the predictive power of CA125 followup certainly reduces the need for regular abdominopelvic scans ${ }^{[16]}$. However, while normal levels of CA125 are reassuring for the patient and her doctor, their measurement often evokes a period of anxiety. In approximately $20 \%$ of patients, serum CA125 levels are not elevated. The majority of these patients have mucinous tumors. In such cases, other 
tumor markers, such as carcinoembryonic antigen or TAG-72,

\section{RESULTS}

The serum levels of HE4, CA-125 and ROMA index in the ovarian cancer group were significantly higher than those in the benign tumor and healthy control groups, and there was significant difference $(\mathrm{P}<0.05)$. The expression level of HE4 and ROMA index in the benign tumor group was not significantly different. The expression level of CA-125 in serum was significantly higher than that in the healthy control group $(\mathrm{P}<0.05$, Table $\mathrm{I})$. The patients in the ovarian benign disease and healthy control groups were further divided into the pre- and postmenopausal groups. The patients with ovarian cancer were divided into the preand postmenopausal groups. The serum levels of HE4, CA-125 and ROMA index were detected to evaluate the sensitivity, specificity, positive predictive value and negative predictive value of HE4, CA-125 and ROMA standardized with pathological diagnosis (Table II). The ROMA index, and a comparison of the sera levels of CA-125 and HE4 in the diagnosis of ovarian cancer in each group indicated significant differences between the three groups $(\mathrm{P}<0.001$, Table III). The AUC of ROC of the ROMA index, HE4 and CA-125 in the diagnosis of ovarian cancer gradually decreased to 0.994, 0.990 and 0.941 , respectively.

\section{DISCUSSION}

The early diagnosis of ovarian malignancies is one of the key factors for improving the survival rate of patients [9]. CA-125 has been used as a tumor marker for the diagnosis and monitoring of ovarian cancer for 30 years, and is also used for efficacy evaluation and monitoring of recurrence [8]. Data have shown that the serum levels of CA-125, HE4 and ROMA in ovarian cancer patients were significantly higher than those of the patients with ovarian benign disease and healthy women [5]. The specificity and positive predictive value of HE4 for ovarian cancer was the highest, and the sensitivity of ROMA index was the highest. In the present study, the 100 cases were divided into the premenopausal and postmenopausal group to evaluate the three indicators in the diagnostic value of ovarian cancer. The ROMA index demonstrated the highest sensitivity and negative predictive value for ovarian cancer. HE4 had the highest specificity and positive predictive value. The specificity of HE4 for ovarian cancer was higher in the postmenopausal women, as reported elsewhere [12]. The sensitivity, specificity, positive predictive value and negative predictive value of the ROMA index in ovarian cancer were the highest (91.89, 96.97, 97.14 and 91.45\%), respectively. CA-125 and HE4 were significantly different from the ROMA index, and the ROMA index was significantly better than CA-125 and HE4 in the diagnosis of ovarian cancer. In addition, the ROC curve drawn in this study for the benign tumor of ovary and healthy control groups identified that the area under the ROC curve of CA-125, HE4 and ROMA index was increased by $0.941,0.990$ and 0.994 , respectively. This result confirmed the clinical diagnostic value of the ROMA index [5]. It also showed that detection of ROMA index in the diagnosis of ovarian cancer was higher than CA125 and HE4.

Table-1: Sera levels of he4, ca-125 and roma index of three groups. Sensitivity $\&$ specificity of positive $\&$ negative predictive values of the $\$$, ca125 and roma \& risk of ovarian cancer algorithm

\begin{tabular}{|l|c|c|c|}
\hline Parameters & Healthy control group & Benign tumor group & Ovarian cancer group \\
\hline Cases & 30 & 64 & 64 \\
\hline HE4 & $39.04 \pm 8.38$ & $54.76 \pm 42.35$ & $739.03 \pm 860.04^{\mathrm{a}}, \mathrm{b}$ \\
\hline CA-125 & $15.08 \pm 5.28$ & $49.07 \pm 175.61^{\mathrm{a}}$ & $868.85 \pm 1204.08^{\mathrm{a}}$, \\
\hline ROMA index & $6.18 \pm 2.21$ & $10.15 \pm 11.98$ & $76.30 \pm 28.57^{\mathrm{a}}, \mathrm{b}$ \\
\hline
\end{tabular}

Table -2: Diagnostic Values of Ca-125, He4 and Roma in Ovarian Cancer

\begin{tabular}{|l|l|l|l|l|}
\hline Characteristics & Sensitivity (\%) & Specificity (\%) & Positive predictive value (\%) & Negative predictive value (\%) \\
\hline CA-125 & $85.07(57 / 64)$ & $92.31(84 / 94)$ & $90.6(57 / 67)$ & $89.36(84 / 91)$ \\
\hline HE4 & $75(48 / 64)$ & $97.87(92 / 94)$ & $96(48 / 50)$ & $85.19(92 / 108)$ \\
\hline ROMA index & $93.75(60 / 64)$ & $92.55(87 / 94)$ & $89.55(60 / 67)$ & $86.14(87 / 101)$ \\
\hline CA-125 & $85.07(57 / 64)$ & $92.31(84 / 94)$ & $90.6(57 / 67)$ & $89.36(84 / 91)$ \\
\hline
\end{tabular}

Table-3: Depicts premenopausal data

\begin{tabular}{|l|l|l|l|l|}
\hline PREMENOPAUSAL & Sensitivity (\%) & Specificity (\%) & Positive predictive value (\%) & Negative predictive value (\%) \\
\hline CA-125 & $92.59(25 / 27)$ & CA-125 & $92.59(25 / 27)$ & CA-125 \\
\hline HE4 & $70.37(19 / 27)$ & HE4 & $70.37(19 / 27)$ & HE4 \\
\hline ROMA index & $96.3(26 / 27)$ & ROMA index & $96.3(26 / 27)$ & ROMA index \\
\hline
\end{tabular}

Table-4: Depicts postmenopausal data

\begin{tabular}{|c|c|c|c|c|}
\hline POSTMENOPAUSAL & Sensitivity (\%) & Specificity (\%) & Positive predictive value (\%) & Negative predictive value (\%) \\
\hline CA-125 & $86.49(32 / 37)$ & CA-125 & $86.49(32 / 37)$ & CA-125 \\
\hline HE4 & $78.38(29 / 37)$ & $96.97(32 / 33)$ & $96.67(29 / 30)$ & $80.00(32 / 40)$ \\
\hline ROMA index & $91.89(34 / 37)$ & $96.97(32 / 33)$ & $97.14(34 / 35)$ & $91.43(32 / 35)$ \\
\hline
\end{tabular}




\section{CONCLUSION}

HE4 and ROMA index which reference intervals are established according to the menopausal status have important clinical significance in the diagnosis of ovarian cancer. Regular detection of serum HE4, CA125, and ROMA index can help predict postoperative recurrence of ovarian cancer. Serological markers provide a means of monitoring tumor activity at many stages of the disease-diagnosis, therapy, and relapse. However, it is important that they are used appropriately and their significance is understood. Knowledge about raised levels of CA125 often raises questions as well as answers; we need to be able to make use of the information available. Early knowledge about relapse does not necessarily help outcome, as better therapies are needed. Progress in therapy is likely to come from a combination of better drugs and a greater understanding of the biology of the disease. Study of serological and tumor-related surface markers needs to continue. Markers for ovarian cancer, and, in particular, CA125, have led the way for epithelial tumors and provide a valuable model for further studies.

\section{REFERENCES}

1. Smith, L. H., \& Oi, R. H. (1984). Detection of malignant ovarian neoplasms: a review of the literature. I. Detection of the patient at risk; clinical, radiological and cytological detection. Obstetrical \& gynecological survey, 39(6), 313-328.

2. Zhang, Z., Bast, R. C., Yu, Y., Li, J., Sokoll, L. J., Rai, A. J., ... \& Berchuck, A. (2004). Three biomarkers identified from serum proteomic analysis for the detection of early stage ovarian cancer. Cancer research, 64(16), 5882-5890.

3. Heintz, A. P. M., Odicino, F., Maisonneuve, P., Quinn, M. A., Benedet, J. L., Creasman, W. T., \& Beller, U. (2006). Carcinoma of the fallopian tube. International Journal of Gynecology \& Obstetrics, 95(S1).

4. Enakpene, C. A., Omigbodun, A. O., Goecke, T. W., Odukogbe, A. T., \& Beckmann, M. W. (2009). Preoperative evaluation and triage of women with suspicious adnexal masses using risk of malignancy index. Journal of Obstetrics and Gynaecology Research, 35(1), 131-138.

5. Karlsen, M. A., Sandhu, N., Høgdall, C., Christensen, I. J., Nedergaard, L., Lundvall, L., ... \& Laursen, I. A. (2012). Evaluation of HE4, CA125, risk of ovarian malignancy algorithm (ROMA) and risk of malignancy index (RMI) as diagnostic tools of epithelial ovarian cancer in patients with a pelvic mass. Gynecologic oncology, 127(2), 379-383.

6. Folk, J. J., Botsford, M., \& Musa, A. G. (1995). Monitoring cancer antigen 125 levels in induction chemotherapy for epithelial ovarian carcinoma and predicting outcome of second-look procedure. Gynecologic oncology, 57(2), 178-182.

7. Urban, N., McIntosh, M. W., Andersen, M., \& Karlan, B. Y. (2003). Ovarian cancer screening. Hematology/oncology clinics of North America, 17(4), 989-1005.

8. Jacobs, I., \& Bast Jr, R. C. (1989). The CA 125 tumour-associated antigen: a review of the literature. Human reproduction, 4(1), 1-12.

9. Moore, R. G., Brown, A. K., Miller, M. C., Badgwell, D., Lu, Z., Allard, W. J., ... \& Lu, K. (2008). Utility of a novel serum tumor biomarker HE4 in patients with endometrioid adenocarcinoma of the uterus. Gynecologic oncology, 110(2), 196201.

10. Levanon, K., Crum, C., \& Drapkin, R. (2008). New insights into the pathogenesis of serous ovarian cancer and its clinical impact. Journal of Clinical Oncology, 26(32), 5284.

11. Holcomb, K., Vucetic, Z., Miller, M. C., \& Knapp, R. C. (2011). Human epididymis protein 4 offers superior specificity in the differentiation of benign and malignant adnexal masses in premenopausal women. American journal of obstetrics and gynecology, 205(4), 358-e1.

12. Hamed, E. O., Ahmed, H., Sedeek, O. B., Mohammed, A. M., Abd-Alla, A. A., \& Ghaffar, H. M. A. (2013). Significance of HE4 estimation in comparison with CA125 in diagnosis of ovarian cancer and assessment of treatment response. Diagnostic pathology, 8(1), 11.

13. Kadija, S., Stefanovic, A., Jeremic, K., Radojevic, M. M., Nikolic, L., Markovic, I., \& Atanackovic, J. (2012). The utility of human epididymal protein 4, cancer antigen 125, and risk for malignancy algorithm in ovarian cancer and endometriosis. International Journal of Gynecologic Cancer, 22(2), 238-244.

14. Sandri, M. T., Bottari, F., Franchi, D., Boveri, S., Candiani, M., Ronzoni, S., \& Sideri, M. (2013). Comparison of HE4, CA125 and ROMA algorithm in women with a pelvic mass: correlation with pathological oncology, 128(2), 233-238.

15. Moore, R. G., Jabre-Raughley, M., Brown, A. K., Robison, K. M., Miller, M. C., Allard, W. J., \& Skates, S. J. (2010). Comparison of a novel multiple marker assay vs the Risk of Malignancy Index for the prediction of epithelial ovarian cancer in patients with a pelvic mass. American journal of obstetrics and gynecology, 203(3), 228-e1.

16. Park, Y., Kim, Y., Lee, E. Y., Lee, J. H., \& Kim, H. S. (2012). Reference ranges for HE4 and CA125 in a large Asian population by automated assays and diagnostic performances for ovarian cancer. International journal of cancer, 130(5), 1136-1144. 